\title{
Invariant Illumination Face Recognition Using Optimized PNN Topology through Neuron Orthogonalization and Genetic Algorithm
}

\author{
Benyamin Kusumoputro and Lina
}

\begin{abstract}
The development of face recognition system attracted significant research attention due to increasing demand on its applications. However, the process of converge to a conclusion of a known-face based from a 2D incoming face images is very difficult, especially when large illumination variations are present in the input space. In this paper, we implemented the illumination compensation praprocessing system in conjuction with the optimized-Probabilistic Neural Networks as a classifier. PNN has shown marvelous higher recognition capability, however, determining the best tuning parameter is very difficult. Neural topology is firstly determined by looking for the most representative neuron using Orthogonal Algorithm, followed by evolutionary determine the best smoothing parameter through Genetic Algorithm. Experiments are conducted using face images under various illumination conditions, and results are presented which illustrate the potential of this approach.
\end{abstract}

Index Terms-Probability neural networks, genetic algorithm, orthogonal algorithm, illumination compensation, face recognition.

\section{INTRODUCTION}

Human has an ability to remember and identify hundreds even thousands of faces whom they meet in their social lifes. The ability in recognizing those faces still can work well although the faces have changes in certain level; such as age, expressions, illumination and addition of accessories. Machine recognition of human face has been developed then, to mimick the ability of human to recognize face. The development of automatic face recognition (AFR) has attracted significant research attention due to increasing demand on its applications. Since AFR is considered to be a natural, non-intimidating, and widely accepted biometric identification method [1], [2], it has the potential of becoming the leading biometric technology. Unfortunately, it is also one of the most difficult pattern recognition problems. So far, all existing solutions provide only partial, and usually unsatisfactory, answers to the market needs, especially illumination variations and partial occlusion of three-dimensional face recognition.

Human visual system could ignored the illumination

Manuscript received October 30, 2013; revised January 23, 2014.

B. Kusumoputro is with the Electrical Engineering Department, Universitas Indonesia, Depok Campus, Depok Indonesia (e-mail: kusumo@ee.ui.ac.id).

Lina is with the Department of Computer Science, Faculty of Information Technology, Tarumanagara University, Jakarta, Indonesia (e-mail: lina@untar.ac.id). variation on the face while recognizing a person, however, the performance of automatic face recognition system (AFR) decrease significantly when various illumination conditions are present in the input space [3]. Therefore, various attempts should be made to handle this issue. Chen et al. [4] then proposed an illumination normalization approach to remove the illumination variations while keeping the main facial features remain. This approach is accomplished by truncating the low-frequency discrete cosine transform (DCT) coefficients in the logarithm domain.

Probabilistic Neural Network (PNN) has also received considerable attention since it shows many successful applications. As one of the promising neural networks system, PNN shown its superior lower computational cost compare with that of back-propagation neural system [5], [6], however, it has a drawback as the number of its hidden neuron increased as much as the learning examples. In PNN [7], [8], every new training data will be represented by a new neuron in the pattern layer, and the network size will increase according to the increment of the used training data, which increased the computation cost of the system. Another problem on using PNN is the difficulty on determining the exact value of the smoothing parameter, where the optimal value depends directly on the characteristics of the known training data. The two problems mentioned above are well realized by researchers and some algorithms are proposed to reduce the training sample, such as the used of vector quantization method for grouping the training sample to find the cluster centers [9]-[11]. Mao et al. [12] have then developed a reduction structure of the PNN by directly used in the selection of the neurons in which at the same time also looking for the best smoothing parameter by using Genetic Algorithms [13]. In attemp to speed up the GA process, we then conducted the best smoothing parameter using different GA technique [14].

In this paper an experimental set up for characteristics performance comparison of the optimized-PNN with that of the standard PNN as a neural classifier in the Face Recognition System are conducted. As the face images are taken with various illumination intensities, illumination normalization is performed prior to discriminate the face through optimized-PNN. Experimental results show that the structured-optimized PNN performed higher recognition rate compare with that of Standard PNN, even using lower number of neurons, which decreased also the total computational cost. Performance comparison of the Optimized-PNN with conventional GA and Decimal-Ga is also conducted and experiment results are carefully studied. 


\section{NEURAL STRUCTURE OPTIMIZATION OF PNN}

\section{A. Structure Determination Using Orthogonal Algorithm}

The main goal of the Orthogonal Algorithm (OA) is to select the most representative neurons in pattern layer of the PNN in order to construct an optimal pattern layer. And as a concequence, higher recognition rate and lower computational cost could be expected by using minimum size of the optimized PNN topology. Probabilistic Neural Network (PNN) is constructed by 4 layers of neurons, i.e. an input layer, a pattern layer, a summation layer, and a decision layer. In PNN learning algorithm, if a vector $x_{i k}$ is a training vector of $k^{\text {th }}$ neuron of class $C_{\mathrm{i}}$, then the maximum probability of vector $x_{i k}$ to be classified as member of $C_{i}$ is:

$$
p_{i}\left(x_{i k}\right)=\sum_{j=1}^{N_{i}} \varphi_{i j}\left(x_{i k}\right)
$$

where

$$
\varphi_{i j}\left(x_{i k}\right)=\frac{1}{(2 \pi)^{d / 2} \sigma^{d}} \frac{1}{N_{i}} \exp \left[-\frac{\left(x_{i k}-x_{i j}\right)^{T}\left(x_{i k}-x_{i j}\right)}{2 \sigma^{2}}\right]
$$

with $p_{i}\left(x_{i k}\right)$ is a nonlinear function of $\sigma$ and vector $x_{i k}$. But if value of $\sigma$ had been determined before and output of each neuron $\phi_{i j}\left(x_{i j}\right)$ is treated as a variable, then $p_{i}\left(x_{i k}\right)$ will become a linier combination of $\phi_{i j}\left(x_{i j}\right)$ as shown in (3). These variables will then be used to evaluate the degree of importance of every neuron in pattern layer.

Equation (1) can also be written in matrix form as $P=\Phi \theta$, with

$$
\begin{gathered}
\theta=[1,1, \ldots, 1]^{T} \\
P=\left[p_{i}\left(x_{i 1}\right), p_{i}\left(x_{i 2}\right), \ldots, p_{i}\left(x_{i N_{i}}\right)\right]^{T} \\
\Phi=\left[\begin{array}{cccc}
\varphi_{i 1}\left(\mathrm{x}_{\mathrm{i} 1}\right) & \varphi_{i 2}\left(x_{i 1}\right) & \ldots & \varphi_{i N_{i}}\left(x_{i 1}\right) \\
\varphi_{i 1}\left(\mathrm{x}_{\mathrm{i} 2}\right) & \varphi_{i 2}\left(x_{i 2}\right) & \ldots & \varphi_{i N_{i}}\left(x_{i 2}\right) \\
\ldots & \ldots & \ldots & \ldots \\
\varphi_{i 1}\left(x_{i N_{i}}\right) & \varphi_{i 2}\left(x_{i N_{i}}\right) & \ldots & \varphi_{i N_{i}}\left(x_{i N_{i}}\right)
\end{array}\right]
\end{gathered}
$$

Transforming matrix $\Phi$ using orthogonal transformation will give,

$$
\Phi=\mathrm{QR}=\left[Q_{1}, Q_{2}, \ldots, Q_{N i}\right] R
$$

where $Q_{1}, Q_{2}, \ldots, Q_{N i}$ are an orthogonal basis and $R$ is an upper triangular matrix. Degree of importance $\left(\Gamma_{j}\right)$ of $j^{\text {th }}$ candidate neuron that member of class $C_{i}$ is calculated based on norm of vector $Q_{j}$, i.e.

$$
\Gamma_{j}=Q_{j}^{T} Q_{j}
$$

In condition where all neurons have the same smoothing parameter value, the higher value of $\left(\Gamma_{j}\right)$ showing the more important the $j^{\text {th }}$ neuron is.

The determination procedure on looking for the most important neuron can be summerized as follows:

1) Choose the most representative neuron from $N_{i}$ neuron of class $C_{i}$ by searching neuron that has highest degree of importance. Then use selected neuron to calculate $Q_{l}$.

$$
\begin{gathered}
Q_{1}^{(\alpha)}=\varphi_{\alpha}, \quad \alpha=1,2, \ldots, N_{i} \\
\phi_{\alpha}=\left[\phi_{\alpha}(1), \phi_{\alpha}(2), \ldots, \phi_{\alpha}\left(\mathrm{N}_{\mathrm{i}}\right)\right]^{T}
\end{gathered}
$$

Degree of importance is calculated as,

$$
\Gamma_{1}^{(\alpha)}=\left[Q_{1}^{(\alpha)}\right]^{T} Q_{1}^{(\alpha)}, \quad \alpha=1,2, \ldots, N_{i}
$$

2) Choose $j^{\text {th }}$ most representative neuron from remaining neurons (total remaining neurons $=N_{i}-j+1$ ). Neuron has highest degree of importance is then selected as $j^{\text {th }}$ most representative neuron.

$$
\begin{gathered}
\mathrm{Q}_{\mathrm{j}}^{(\alpha)}=\phi_{\mathrm{k}_{\alpha}}-\sum_{\mathrm{l}=1}^{\mathrm{j}-1} \mathrm{r}_{l \alpha}^{(\alpha)} \mathrm{Q}_{l}, \alpha=1,2 \ldots, N_{i}-j+1 \\
\mathrm{r}_{l \alpha}^{(\alpha)}=\mathrm{Q}_{l}^{\mathrm{T}} \phi_{\mathrm{k}_{\alpha}} / \mathrm{Q}_{l}^{\mathrm{T}} \mathrm{Q}_{l} \quad, \alpha=1,2, \ldots, N_{i}-j+1, l<i
\end{gathered}
$$

\section{B. Smoothing Parameter Determination Using Genetic Algorithm}

In PNN, the determination of the smoothing parameter is a critical aspect, due to its direct connection with the recognition accuracy of the neural networks system. An appropriate smoothing parameter is often data dependent; therefore the selection of the smoothing parameter is an essential step to be taken in every problem that uses PNN as the classifier. Suppose we already have the network size of the PNN, with $n$ is the number of selected neuron in pattern layer, then the problem can be defined as a constrained optimization problem of $\min \{n\}$ subject to:

$$
\eta<\delta
$$

where $\eta$ is error rate of classification, and $\delta$ is a given maximum limit of error rate tolerance of classification. Since quantitative relation between network size, error rate of classification, and smoothing parameter $(\sigma)$ is not exist, then genetic algorithm (GA) is used to solve above optimization problem.

GA is a searching algorithm, which developed based on nature selection of genetics. Evolutionary computation of GA searching algorithm is accomplished by applying GA's processes, i.e. encoding, fitness value evaluation, reproduction, crossover, and mutation. In this paper, we used a decimal Genetic Algorithm proposed in [14]. Instead of using string or binary number as a representation of the chromosomes, in this technique the chromosomes are represented directly using real number of smoothing parameter. Overall GA approach is then completedly different with that of [13], and proofed to be very effective in decreasing the computational cost of the GA. The evaluation 
of the best chromosome, or the objective fitness function, is performed by using the optimal recognition rate of the training data and the testing data for training (as part of the data training). As already explained earlier, every individual represents a smoothing parameter value, and by using every possible value of $\sigma$, a set of network structure candidates are determined as the chromosomes.

The selection process is evaluated by Roullete Wheel, where the chromosomes with the optimal fitness value have a better possibility to be determined as the winner. The crossover operator then is used using:

$$
\begin{gathered}
X_{p}=\frac{1}{4}\left(3 X_{p}+Y_{p}\right) \\
Y_{p}{ }^{\prime}=\frac{1}{2}\left|2 Y_{p}-X_{p}\right|
\end{gathered}
$$

where $X_{p}$ and $Y_{p}$ the parents chromosomes and $X_{p}$ ' and $Y_{p}$ ' the offsprings chromosomes.

\section{FACE RECOGNITION UNDER VARIOUS ILLUMINATION CONDITIONS}

Illumination normalization and compensation approach is developed for removing the illumination variations that may occur between the individual face images taken from real camera surveillance, for instance, with all the templates face images stored in the gallery database. Chen et. al [4] then proposed an illumination normalization approach to remove the illumination variations while keeping the main facial features remain. This approach is accomplished by truncating the low-frequency discrete cosine transform (DCT) coefficients in its logarithm domain.

Suppose an image gray level $f(x, y)$ is defined as

$$
f(x, y)=r(x, y) \cdot e(x, y)
$$

where $r(x, y)$ the reflectance, and $e(x, y)$ the illumination [15]. By taking the logarithm transform on (11) we have

$$
\log f(x, y)=\log r(x, y)+\log e(x, y)
$$

Suppose we have new images with different illumination $f^{\prime}(x, y)$, and by comparing in logarithm form such as in (12), then we have

$$
\log f^{\prime}(x, y)=\log r(x, y)+\log e^{\prime}(x, y)
$$

Since the reflectance $r(x, y)$ is supposed to be stable characteristic of the facial features, the changing illumination effect of the image could be compensated by $\in(x, y)$, in the form of

$$
\log f^{\prime}(x, y)=\log r(x, y)+\log e(x, y)-\in(x, y)
$$

$$
\log f^{\prime}(x, y)=\log f(x, y)-\in(x, y)
$$

Suppose we have a $M x N$ image $F(x, y)$ in logarithm domain that in the DCT frequency domain can be written as

$$
\begin{aligned}
& C(u, v)=\alpha(u) \alpha(v) \times \\
& \sum_{x=0}^{M-1} \sum_{y=0}^{N-1} F(x, y) \cos \left[\frac{\pi(2 x+1) u}{2 M}\right] \cos \left[\frac{\pi(2 y+1) v}{2 N}\right]
\end{aligned}
$$

And the inverse transform is defined as

$$
F(x, y)=\sum_{u=0}^{M-1} \sum_{v=0}^{N-1} E(x, y)
$$

With

$$
\begin{aligned}
& E(x, y)=\alpha(u) \alpha(v) C(u, v) x \\
& \cos \left[\frac{\pi(2 x+1) u}{2 M}\right] \cos \left[\frac{\pi(2 y+1) v}{2 N}\right]
\end{aligned}
$$

And

$$
\alpha(u)=\left\{\begin{array}{l}
\frac{1}{\sqrt{M}}, u=0 \\
\sqrt{\frac{2}{M}}, u=1,2, \ldots, M-1
\end{array} \text { and } \alpha(v)=\left\{\begin{array}{l}
\frac{1}{\sqrt{N}}, v=0 \\
\sqrt{\frac{2}{N}}, v=1,2, \ldots, N-1
\end{array}\right.\right.
$$

As the illumination variations are mainly in the low frequancy domain, a new image with different illumination condition in logarithm domain in DCT domain can be written as

$$
F^{\prime}(x, y)=\sum_{u=0}^{M-1} \sum_{v=0}^{N-1} E(u, v)-\sum_{j=1}^{J} E\left(u_{j}, v_{j}\right)
$$

With the $i$ low-frequency DCT coefficients can be extracted as

$$
\varepsilon(u, v)=\sum_{j=1}^{J} E\left(u_{j}, v_{j}\right)
$$

The DCT coefficient which is related with the uniform illumination can be setting to the same value as $C(0,0)=\log \mu \sqrt{M N}$. And by using this coefficients as the reference, the low-frequency DCT coefficient to be discarded is determined.

In this paper, we have used the University of Indonesia Computational Intelligence Lab (UICIL) database. The database contains images of ten individual of Indonesian persons under six various illumination conditions, four different expressions (neutral, angry, laugh, and smile). The database consist of a total of 400 images and we devided into $50 \%$ for tranining the neural networks and the rest is used for testing phase.

Fig. 1 shows the intial face images and its reconstructed after passing the illumination compensation processes with $\log \mu=150, M=N=30$. Generally, the face recognition using appearance-based approaches deal with a set of learning images taken at predetermined pose position with various capturing conditions. Since these images are usually 
high-dimensional images, they could not be applied directly due to efficiently reasons. Hence, a transformation to Eigen space, in which its dimensionality is much lower than that in the original image-space, is usually utilized. PCA is an efficient transformation procedure to represent a collection by mapping the original $n$-dimensional space onto a $k$-dimensional feature subspace where normally $k \ll M \times N$. Since the PCA transformation process has almost used for dimensionality reduction purpose in appearance based face recognition system, this eigenspace transformation is not explained here. Interested readers may refer to [16] for more detailed information of the transformation process.

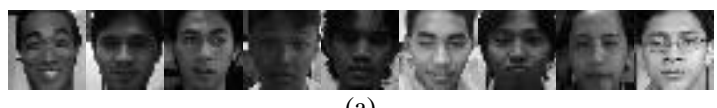

(a)

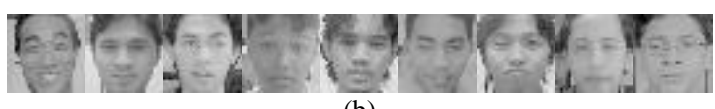

(b)

Fig. 1. Comparison of the face images before and after illumination compensation processes. (a) face images taken with different illumination conditions (b) reconstructed of face images after illumination compensation processes.

\section{EXPERIMENTS AND RESULTS}

Experiments are conducted using a training/testing paradigm of 50:50 percentage. However, in the training phase, we have devided further the learning algorithm into two phases, i.e. Phase1 $(P \# 1)$ and Phase2 (P\#2), respectively. In Phase1, the learning data is intended for PNN structure determination, while in Phase2, the learning data is used for GA optimization process. We have then three training datasets, i.e., DataSet\#1 that consists of $20 \%$ P\#1 and 30\% P\#2, DataSet\#2 that consists of 30\% P\#1 and 20\% P\#2, and

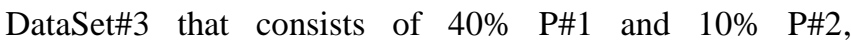
respectively. We have not used $10 \% \mathrm{P \# 1}$, since this data set is too small for training the PNN. Experiments are conducted five times, consecutively, and the average recognition is calculated and depicted in the table of results.

Table I shows the recognition rate of the optimized-PNN without illumination compensation processes. As we can see from this table, the overall recognition rate of the system is high enough, showing that the characteritics of the optimized-PNN using orthogonal algorithms and genetic algorithms are performed quite optimal.

However, when the neural system used DataSet\#1, the recognition rate is lower compare with that of using DataSet\#2 and DataSet\#3, respectively. This lower recognition rate show that the active neurons in the hidden layer may not covered all the most information in the overall data set.

TABLE I: RECOGNITION RATE OF THE OPTIMIZED-PNN FOR FACE IMAGES WITHOUT ILLUMINATION COMPENSATION PROCESSES

\begin{tabular}{lllllll}
\hline \hline Training Data & \multicolumn{6}{c}{ Recognition Rate (\%) } \\
Set (\%) & 1 & 2 & 3 & 4 & 5 & Average \\
\hline & & & & & & \\
DataSet\#1 & 94 & 93 & 95 & 94 & 93 & 93.6 \\
DataSet\#2 & 95 & 96 & 95 & 96 & 95 & 95.4 \\
DataSet\#3 & 95 & 95 & 96 & 95 & 95 & 95.2 \\
& & & & & & \\
\hline \hline
\end{tabular}

TABLE II: RECOGNITION RATE OF THE OPTIMIZED-PNN USING CONVENTIONAL GA FOR FACE IMAGES WITH ILLUMINATION COMPENSATION PROCESSES

\begin{tabular}{lllcccc}
\hline \hline Training Data & \multicolumn{6}{c}{ Recognition Rate (\%) } \\
Set (\%) & 1 & 2 & 3 & 4 & 5 & Average \\
\hline & & & & & & \\
DataSet\#1 & 96 & 96 & 96 & 96 & 96 & 96.0 \\
DataSet\#2 & 98 & 98 & 98 & 98 & 98 & 98.0 \\
DataSet\#3 & 98 & 98 & 98 & 98 & 98 & 98.0
\end{tabular}

TABLE III: RECOGNITION RATE OF THE OPTIMIZED-PNN USING DECIMAL GA FOR FACE IMAGES WITH ILLUMINATION COMPENSATION PROCESSES

\begin{tabular}{ccccccc}
\hline \hline Training Data & \multicolumn{6}{c}{ Recognition Rate (\%) } \\
Set (\%) & 1 & 2 & 3 & 4 & 5 & Average \\
\hline & & & & & & \\
DataSet\#1 & 96 & 97 & 96 & 97 & 96 & 96.4 \\
DataSet\#2 & 99 & 98 & 98 & 98 & 98 & 98.2 \\
DataSet\#3 & 98 & 98 & 98 & 98 & 98 & 98.0 \\
& & & & & & \\
\hline \hline
\end{tabular}

Tabel III shows the recognition rate of optimized-PNN using conventional GA and with illumination compensation processes. It is clearly seen from this table, the recognition rate of the system is higher compare with that in Table I, for all of the three data sets. As also can be seen from this table, the recognition rate for all five concecutive experiment show nearly the same, means that the system has a very stable output characteristics. These increment of recognition rate and stability characteristic show that the illumination compensation has an important impact on increasing the capability of the developed system.

Table III shows the recognition rate of the PNN system as used for the experiments depicted in Table II, however, in these experiments we have used decimal GA instead of the conventional GA. As can be seen from this table, the recognition rate for all of the data sets are comparable with that of in Table II, with just a slide increment. This comparison shows that the numerical GA has a comparable performance on determining the best smoothing parameter of the optimized-PNN.

TABLE IV: COMPARISON OF THE RECOGNTION RATE OF THE THREE OPTIMIZED-PNN FOR VARIOUS TRAINING AND TESTING DATA SETS Recognition Rate (\%)

\begin{tabular}{ccccccc} 
Training Data & \multicolumn{3}{c}{ OGA-PNN w/o } & \multicolumn{3}{c}{ OGA-PNN with OGA*-PNN with } \\
Set (\%) & \multicolumn{2}{c}{ IC } & \multicolumn{2}{c}{ IC } & \multicolumn{2}{c}{ IC } \\
& Train & Test & Train & Test & Train & Test \\
\hline & & & & & & \\
DataSet\#1 & 90.0 & 93.6 & 90.0 & 96.0 & 94.0 & 96.4 \\
DataSet\#2 & 100 & 95.4 & 100 & 98.0 & 100 & 98.2 \\
DataSet\#3 & 100 & 95.2 & 100 & 98.0 & 100 & 98.0
\end{tabular}

OGA-PNN: Optimized-PNN using conventional GA

OGA*-PNN: Optimized-PNN using decimal GA

IC: Illumination compensation processes

Table IV shows the results comparison of the three Optimized-PNN systems, i.e. Optimized-PNN using conventional GA without illumination compensation, Optimized-PNN using conventional GA with illumination compensation, and Optimized-PNN using decimal GA with illumination compensation. Performance comparison is 
performed and compared in terms of the average recognition rate for data training and data testing, respectively, using the three data sets previously defined. As can be seen from this table, the recognition rate of all the three optimized-PNN are around $90 \%$ when using DataSet\#1. This recognition rate are much lower compare with that of using DataSet\#2 and DataSet\#3, respectively, that can reach $100 \%$. This results show that the DataSet\#1 is not properly enough, and as the consequence, the recognition rate of the system is also lower. It is also confirmed by these experiments that using DataSet\#2 and DataSet\#3, the recognition rate of the testing face images are high enough. It can be seen also that the used of decimal GA, however, could not increase the recognition rate of the Optimized-PNN considerably.

TABLE V: COMPARISON OF THE COMPUTATIONAL TRAINING TIME AND THE ACTIVE NEURONS OF THE THREE OPTIMIZED-PNN

\begin{tabular}{ccccccc}
\hline \hline $\begin{array}{c}\text { Training } \\
\text { Data Set } \\
(\%)\end{array}$ & \multicolumn{2}{c}{ OGA-PNN w/o } & \multicolumn{2}{c}{ OGA-PNN with } & \multicolumn{2}{c}{ OGA*-PNN } \\
Time & Neuron & Time & Neuron & Time & Neuron \\
\hline & & & & & & \\
DataSet\#1 & 40.3 & $85 \%$ & 3.9 & $65 \%$ & 1.2 & $65 \%$ \\
DataSet\#2 & 41.3 & $80 \%$ & 10.6 & $46 \%$ & 1.1 & $45 \%$ \\
DataSet\#3 & 41.7 & $80 \%$ & 2.48 & $29 \%$ & 1.1 & $29 \%$
\end{tabular}

OGA-PNN: Optimized-PNN using conventional GA

OGA*-PNN: Optimized-PNN using decimal GA

IC: Illumination compensation processes

Table V shows the results comparison of the three Optimized PNN system in terms of the computational cost (second) and the active neurons in the PNN topology. As can be seen from this table, the percentage of the active neurons of the Optimized-PNN without illumination compensation processes are about 85 to $80 \%$, much more higher than that of the Optimized-PNN when using illumination compensation processes. These results show that the illumination compensation processes has increased the performance of the Optimized-PNN considerably, and when using proper learning data set, the active neurons could be reduced significantly, with only $29 \%$ of the overall neurons. It is clearly seen also from this table that the used of decimal GA have nearly the same percentage of active neurons as with that conventional GA.

However, as can be seen in Table V, the computational cost of the Optimized-PNN using decimal GA are much lower than that of Optimized-PNN using conventional GA, showing the characteritic superiority of the decimal GA compare with that of conventional GA.

\section{CONCLUSION}

We have developed and implemented the face recognition system based on optimized PNN through Orthogonal and Genetic Algorithms in conjuction with an illumination compensation processes as a data preprocessing system. It is proofed from the experiments that the Optimized-PNN through Orthogonal algorithm for minimizing the neuron topology and GA for searching the optimal smoothing parameter have improved the recognition capability of the classifier system. It is also shown that the illumination compensation preprocessing system also very important in improving the system performance, especially when using decimal GA. By normalizing the illumination variation, the number of active neurons could be decreased significantly, which as a consequence decreasing further the learning time. It is also proved that the utilization of illumination compensation processes increases the recognition capability of the Optimized-PNN considerably. It is hoped that higher increment of the recognition rate could be achieved when the system is used for three-dimensional face recognition system with various pose and more harsh illumination variations.

\section{REFERENCES}

[1] J. Ashbourn, Biometric: Advanced Identity Verification, Springer Verlag: Berlin, Heidelberg, New York, 2002.

[2] J. Ortega-Garcia, J. Bigun, D. Reynolds, and J. G. Rodiguez, "Authentification gets personal with biometrics," IEEE Signal Processing Magazine, vol. 21, no. 2, pp. 50-62, 2004.

[3] W. Zhao, R. Chellapa, P. J. Phillips, and A. Rosenfeld, "Face recognition: a literature survey," ACM Computing Surveys, vol. 35, no. 4, pp. 399-458, 2003.

[4] W. Chen, M. J. Er, and S. Wu, "Illumination compensation and normalization for robust face recognition using discrete cosine transform in logarithm domain," IEEE Trans. Systems, Man, and Cybernetics, B: Cybernetics, vol. 36, no. 2, pp. 458-466, 2006.

[5] B. Kusumoputro and M. Rivai, "Discrimination of fragrance odor by arrayed quartz resonator and a neural network," in Proc. Int. Conf. on Comput. Intelli. and Multimed. Application, 1998, pp. 264-269.

[6] B. Kusumoputro, "Comparison of FALVQ and modified back-propagation in artificial odor discrimination system," in Proc. Int. Conf. on Modelling, Identification and Control, Innsbruck, Austria, February 15-19, 1999, pp. 434-437.

[7] D. F. Specht, "Probabilistic neural networks and the polynomial adaline as complementary techniques for classification," IEEE Trans. on Neural Networks, vol. 1, no.1, pp. 111-121, 1990.

[8] T. Masters, Advanced Algorithms for Neural Networks: A C++ Sourcebook, John Wiley \& Sons, 1995.

[9] P. P. Raghu and B. Yegnanarayana, "Supervised texture classification using a probabilistic neural network and constraint satisfaction model," IEEE Transaction on Neural Networks, vol. 9, no. 3, 1998.

[10] P. Burrascano, "Learning vector quantization for the probabilistic neural networks," IEEE Trans. on Neural Networks, vol. 2, no. 2, pp. 458-461, 1991.

[11] A. Zaknich, "A vector quantization reduction method for the propabilistic neural networks," in Proc. IEEE Int. Conf. Neural Networks, Piscataway, NJ, 1997, pp. 1117 - 1120.

[12] K. Z. Mao, K. C. Tan, and W. Ser, "Probabilistic neural-network structure determination for pattern classification," IEEE Transaction on Neural Networks, vol. 11, no. 4, 2000.

[13] D. E. Goldberg, Genetic Algorithms in Search, Optimization and Machine Learning, Addison Wesley, 1989.

[14] K. K. Yan and T. Lu, "Sidelobe reduction in array-pattern synthesis using GA," IEEE Trans. Antenas Propagat., vol. 45, 1997.

[15] E. H. Land and J. J. McCann, "Lighness and retinex theory," J. Opt. Soc. Amer., vol. 61, pp. 1-11, 1971.

[16] Lina and B. Kusumoputro, "Determination of 3-D image viewpoint using modified nearest feature line method in Its Eigen space domain," WSEAS Trans. on Computers, vol. 2, no. 1, pp. 140-147, 2003.

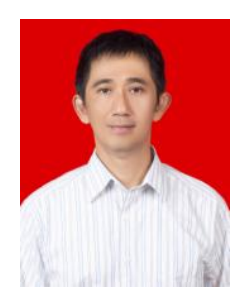

Benyamin Kusumoputro was born in Bandung, West Java, Indonesia on November 17, 1957. He finished his under graduate course from Department of Physics, Bandung Institute of Technology, Bandung, Indonesia in 1981. He then entered Graduate School of Optoelectronics in University of Indonesia, Jakarta, Indonesia under supervision from Tokyo Institute of Technology, Tokyo, Japan, and finished in 1984. From 1984 to 1986 , he worked at Phillips Development Corporation in Jakarta, and moved back to University of Indonesia as a research associate. From 1988 to 1990 he was a research student at Department of Electrical and Electronic Engineering, Graduate 
School of Science and Engineering, Tokyo Institute of Technology, and entered doctoral cource in the field of biosensor and micromachining at the same department under Monbukagakusho Scholarship Program. He was granted a doctor of engineering degree in 1993. He is now a professor of computational intelligence at Department of Electrical Engineering, University of Indonesia. His research of interest including the 3D modelless face recognition system, intelligent control of unmanned aerial vehicle and quality classification of nuclear fuel using computational intelligence methods. He received best researcher award from Indonesia Ministry of Reseach and Technology in 2006.

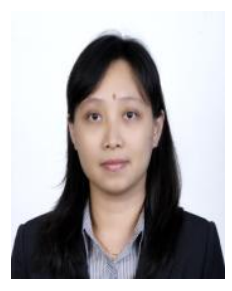

Lina was born in Padang, West Sumatra, Indonesia on February 20, 1979. She finished her under graduate course from Department of Computer Science, Faculty of Engineering, Tarumanagara University, Jakarta, Indonesia in 2001. She then entered the Graduate School of Computer Science at University of Indonesia and graduated in 2004. In 2005 she was a research student at the Department of Media Science, Graduate School of Information Science, Nagoya University, and entered the doctor course in 2006 and granted a $\mathrm{Ph} . \mathrm{D}$ in 2009. She is now a lecturer at the Department of Computer Science, Faculty of Information Technology, Tarumanagara University. Her research interests are in the area of image recognition and intelligent system. 